Relations industrielles

Industrial Relations

\title{
Blue-Collarite, par Arthur B. Shostak, New York, Random \\ House Inc., 1969, 299 pp.
}

\section{André Roy}

Volume 26, numéro 4, 1971

URI : https://id.erudit.org/iderudit/028277ar

DOI : https://doi.org/10.7202/028277ar

Aller au sommaire du numéro

Éditeur(s)

Département des relations industrielles de l'Université Laval

ISSN

0034-379X (imprimé)

1703-8138 (numérique)

Découvrir la revue

Citer ce compte rendu

Roy, A. (1971). Compte rendu de [Blue-Collarite, par Arthur B. Shostak, New York, Random House Inc., 1969, 299 pp.] Relations industrielles / Industrial Relations, 26(4), 1053-1058. https://doi.org/10.7202/028277ar

Tous droits réservés (C) Département des relations industrielles de l'Université Laval, 1971
Ce document est protégé par la loi sur le droit d'auteur. L'utilisation des services d'Érudit (y compris la reproduction) est assujettie à sa politique d'utilisation que vous pouvez consulter en ligne.

https://apropos.erudit.org/fr/usagers/politique-dutilisation/ 


\section{RECENSIONS}

Blue-Collarite, par Arthur B. Shostak, New York, Random House Inc., 1969, 299 pp.

Il s'agit ici d'une étude sociologique assez poussée du col bleu de race blanche de sexe masculin aux Etats-Unis. L'ouvrage se divise en cinq parties.

La première partie s'efforce de situer ce type d'homme dans ses origines démographiques et dans ses avatars historiques, ce que l'auteur appelle son odyssée. C'est, la plupart du temps, un immigrant - sur 33 millions de nouveaux Américains, 60 pour cent appartiennent encore à la première génération - venant principalement d'Italie, d'Allemagne, du Canada, de Pologne, de Grande-Bretagne ou de Russie. Immigrant ou pas, il est fils d'un col bleu ou d'extraction rurale, les statistiques indiquant que le cinquième des ouvriers d'usine sont nés sur une ferme. Il a aussi de bonnes chances d'être catholique et d'être assez peu instruit. Il a également été marqué par les années sombres de la grande dépression. L'ouvrier américain a vécu et continue de vivre avec la hantise d'une nouvelle crise économique entretenue par l'irrégularité de l'emploi, de telle manière que le passé pas si lointain ne lui donne que peu de confiance dans l'avenir.

Cet état d'anxiété et d'insécurité va expliquer dans une certaine mesure son comportement professionnel, familial, social. Mais, au préalable, qu'est-ce qu'un col bleu ? Quel est son statut social dans la réalité ou tel qu'il le perçoit ?

Si l'on commençait par les dénombrer ! Ils sont une trentaine de millions aux Etats-Unis, se répartissant à peu près également entre manoeuvres, ouvriers spécialisés et ouvriers qualifiés, et ces trois blocs ont conservé au cours des quarante dernières années une stabilité assez remarquable, même si la proportion des manoeuvres a tendance à diminuer.

Le travail des premiers n'exige aucune dextérité. Leur tâche est routinière; elle peut souvent s'apprendre en une demiheure le premier jour de leur emploi.
Aux seconds, il faut quelques semaines ou quelques mois pour s'initier au travail et, dans leur cas aussi, il s'agit aussi de besognes routinières. De tous les cols bleus, les ouvriers qualifiés sont beaucoup mieux partagés. Leur tâche exige souvent un long apprentissage, une activité intellectuelle considérable; elle demande de l'imagination, comporte des responsabilités.

Ces différences de statut professionnel entraîne un comportement personnel des individus qui apparaît quelque peu paradoxal. Les manoeuvres sont surtout préoccupés par la question pécuniaire : leurs salaires sont bas par rapport à ceux des autres groupes, mais, occupés surtout dans des emplois saisonniers, la crainte du chômage ne les dérange pas beaucoup, car ils sont habitués à le considérer comme quelque chose qui va de soi. Pour les ouvriers spécialisés, c'est la sécurité de leur emploi qui importe, parce que la perte de leur poste signifie pour eux souvent de longues périodes de chômage. Quant aux ouvriers qualifiés, leur principale préoccupation est la protection d'un métier qui procure un certain sentiment de satisfaction personnelle dans un atelier où ils trouvent appui et support chez leurs compagnons de travail, d'un métier qu'ils ont parfois l'ambition de transmettre à leurs enfants. En fait, sont-ils souvent plus inquiets que les autres devant la multiplication des changements technologiques et du phénomène de l'automation. Contrairement aux manoeuvres et aux ouvriers spécialisés dont le statut professionnel a de bonnes chances de demeurer stable, les ouvriers qualifiés ressentent fortement la tension d'un glissement toujours possible vers une situation moins reluisante.

Mais, au-delà de ces différenciations entre les trois groupes, il y a des facteurs à la fois subjectifs et objectifs qui marquent profondément la masse des cols bleus. Subjectivement, les travailleurs manuels se sentent aliénés dans un statut social qu'ils considèrent eux-mêmes tout au bas de l'échelle et que l'opinion publique percoit ainsi, compte tenu des éléments qui le composent: revenus 
pécuniaires, niveau d'instruction, nature du travail, influence et prestige. Aussi ressentent-ils fortement l'impression d'être dans une situation qui n'est pas conforme au rôle utile qu'ils jouent dans la société.

Leur travail, à cause de sa nature routinière ou parcellaire, dénué souvent de toute responsabilité personnelle, exécuté la plupart du temps bien loin de celui qui en profitera, en vient à manquer complètement de motivation intrinsèque, ce qui engendre des phénomènes psychiques aberrants : sentiments d'agression, refuge dans la rêverie éveillée, construction d'une vie imaginaire hors de toute réalité. Ceci entraîne vis-à-vis les autres groupes sociaux un comportement marqué souvent d'expressions de mépris à l'endroit des cols blancs, d'opposition au travail industriel des femmes mariées en qui ils voient des concurrentes et aussi de forte animosité contre les noirs qu'ils tiennent pour d'éternels enfants.

En outre, cet état d'esprit déjà assez lourd à supporter est intensifié par des facteurs objectifs : manque à gagner, travail effectué dans des conditions pénibles, insécurité de l'emploi. Aux EtatsUnis, en 1965, les ouvriers spécialisés, les employés de service et les manoeuvres se trouvaient respectivement aux trois derniers rangs dans l'échelle des gains annuels. $\mathrm{Ce}$ qui est encore plus symptômatique, près de la moitié des chômeurs se trouvaient dans ces catégories.

Pour lutter contre cet état de choses, le col bleu américain dispose d'un seul instrument: le syndicalisme. L'auteur y consacre quelques pages. Elles sont fort pessimistes. Le syndicalisme manque de plus en plus de mordant; il a perdu l'élan de militantisme qui l'avait marqué au cours des années d'avant-guerre à l'époque du New Deal. Shostak attribue cet amollissement aux facteurs suivants : désintéressement des travailleurs de la base, embourgeoisement des grands syndicats, instauration d'un syndicalisme paternaliste et collaborateur. L'Auteur voit un espoir de réveil au niveau local chez le délégué d'atelier. Mais celui-ci, trop souvent, est porté à voir là un moyen de s'intégrer à l'entreprise, d'aspirer à un poste de contremaître, voire de directeur du personnel. Néanmoins, le syndicalisme, parce qu'il groupe près de la moitié des cols bleus, peut permettre une reprise à condition, pour citer un mot de Walter Reuther, de «syndiquer les syndiqués $\gg$.
Le chapitre consacré au syndicalisme clôt l'étude de Skostak sur la condition professionnelle des travailleurs manuels. Dans la deuxième partie de son ouvrage, il va les regarder vivre dans la société immédiate qui les encadre: avec leurs voisins, à l'intérieur de leur farnille, dans le prolongement de leurs enfants, garçons et filles.

L'Auteur analyse le comportement des cols bleus dans leurs relations de voisinage selon le milieu dans lequel ils vivent : d'abord les quartiers anciens des villes où ils se sont agglomérés par groupes ethniques ou encore suivant leurs croyances religieuses, enclaves où ils luttent pour préserver les caractères de leur origine raciale et de leur culture. Mais, par suite du vieillissement des immeubles, des exigences de la circulation et du stationnement, ces quartiers se détériorent et, malgré des efforts qu'ils savent inutiles, ils se voient graduellement parqués dans des ghettos de plus en plus avilissants au milieu de terrains vagues où s'accumulent débris et ordures de toutes sortes. A l'opposé de ces enclaves, il y a les banlieues ouvrières stables. Elles datent souvent d'assez loin alors que les groupes d'ouvriers spécialisés et qualifiés avaient suivi les usines qui s'étaient installées à la périphérie des villes. A ces endroits, les cols bleus ont pu y créer un milieu humain plus intéressant tout en conservant les symboles valables de leur habitat antérieur. Enfin, il arrive que le col bleu s'installe dans les banlieues où déjà les familles des classes moyennes ont pris pied. Ce n'est généralement pour eux qu'un séjour transitoire. Il se produit un phénomène bizarre : ou les cols bleus s'implantent solidement en ce nouveau milieu et peu à peu les classes moyennes disparaissent; ou, dans le cas contraire, c'est la famille ouvrière qui part. En effet, si la femme, qui est souvent plus instruite, peut s'accomoder de ce voisinage, l'homme ne parvient pas à comprendre ces moeurs différentes.

Dans cet environnement, ce sont des familles qu'on retrouve. Shostak y découvre deux types de foyers, le foyer traditionnel où l'homme et la femme vivent isolés l'un de l'autre dans une division très claire des tâches entre partenaires ainsi que dans la poursuite d'intérêts et d'activités distinctes. Dans ces familles, l'homme est le pourvoyeur et il tire de là toute sa valeur. C'est la 
femme qui élève les enfants, tient les finances domestiques, règle les questions administratives, s'occupe des relations de voisinage et d'affaires. Mais, dans certains groupes, un nouveau type de famille tend à se développer où il $\mathrm{y}$ a beaucoup plus d'intégration entre maris et femmes. Il y a partage des responsabilités entre l'homme et la femme, beaucoup de respect et de considération l'un pour l'autre. Bref, la famille est de toutes les institutions sociales celle où le col bleu concentre davantage son intérêt, celle qui lui apporte le plus de satisfaction, même si, à cause des enfants, elle lui cause souvent certaines inquiétudes.

Le col bleu, à quelque catégorie qu'on réfère, désire pour ses enfants, un sort plus intéressant que le sien. Les enfants, garçons ou filles, l'auteur les classe en trois groupes qu'il appelle les rebelles, les conformistes et les ambitieux. Les rebelles n'acceptent pas la condition de leurs parents, sont de mauvais élèves même s'ils ont du talent. C'est dans leurs rangs que se recrutent généralement la masse des délinquants. Les conformistes, et c'est le plus grand nombre, persistent aussi longtemps qu'ils le peuvent dans leurs études, tout en ne visant pas à dépasser le statut professionnel de leurs parents. Ce qu'ils recherchent avant tout, c'est de s'outiller intellectuellement et techniquement pour être en mesure d'obtenir facilement un emploi et de n'avoir pas à redouter le chômage qui a marqué leur enfance et leur adolescence. Quant aux ambitieux, ils visent à enfoncer les barrières sociales, à se hisser à un rang plus élevé. Ils se recrutent principalement dans les familles où la mère $a$ un niveau de scolarité plus élevé que celui de son mari. Mais ceci ne signifie pas qu'ils en soient plus heureux : ils ont en effet, dès leurs études, à supporter souvent les préjugés des fils de professionnels et, plus tard, à se frayer difficilement un chemin dans des couches sociales qui ne les acceptent pas facilement, lorsque les circonstances ne les ont pas forcés de s'arrêter à mi-chemin, ce qui en fait des mécontents.

Mais le col bleu n'est pas uniquement un travailleur et un père de famille. Il est également un homme qui a une vie personnelle à développer, une santé à conserver, des préoccupations éthiques et religieuses; un citoyen qui participe d'une certaine façon à la vie politique.
Dans la troisième partie de son étude, Shostak le considère à ces différents points de vue où l'on peut retrouver dans la pénombre les répercussions de sa vie professionnelle et familiale.

Voyons d'abord le col bleu dans ses loisirs. La diminution de la semaine de travail a augmenté d'un millier d'heures par année le temps dont il peut disposer librement. A quoi le passe-t-il ? A quelles occupations libres vont ses préférences? Selon l'Auteur, le col bleu consacrerait beaucoup de temps à deux formes de loisirs: la télévision qui, par la nature de sa programmation, apporte des réponses faciles à des questions difficiles, voile les aspects sordides et misérables de la vie réelle et réconforte les déroutés; les visites, parce que c'est dans ces groupes que les liens de parenté demeurent les plus forts et les plus étendus. On en trouve une des manifestations les plus typiques dans le magasinage en famille, où père, mère et enfants ensemble peuvent passer des heures à déambuler dans les allées des supermarchés, à lécher les vitrines à la recherche des meilleures aubaines.

Chez un certain nombre, et le phénomène prend plus d'ampleur à mesure que les conditions économiques le permettront, les sports de la pêche, de la chasse, du camping ainsi que le jardinage deviennent de plus en plus populaires. Beaucoup, parmi les jeunes, jettent leur dévolu sur les automobiles de sport.

D'autre part, beaucoup de cols bleus consacrent le plus clair de leur temps aux spectacles sportifs, ils y assistent ou les suivent assidûment à la télévision qui leur accorde d'ailleurs une place de choix dans sa programmation. Les vedettes deviennent pour eux de véritables idoles, surtout si l'on peut rattacher leur nom à un groupe ethnique comme ce fut le cas, par exemple, pour les Dimaggio parmi les Italiens et les Stan Musial parmi les Polonais.

Le col bleu lit-il? Peu ou pas, sauf les tabloids illustrés, principalement ceux de fin de semaine. Mais, fait surprenant, c'est parmi les cols bleus que les organisations fraternelles, Chevaliers de Colomb, Francs-maçons, Anciens combattants, Ku Klux Klan, recrutent une bonne partie de leurs membres les plus fidèles et les plus convaincus.

Devant ces manifestations, l'Auteur ne se montre pas trop pessimiste : il conclut que, d'une façon générale, l'ouvrier 
choisit librement ses loisirs et que ceux-ci n'exercent qu'une influence plutôt secondaire sur son comportement.

Quel rôle jouent les quelques trente millions de cols bleus aux différents niveaux de la vie politique? Leur vote, quand ils l'exercent, peut faire et défaire les gouvernements. Mais les ouvriers américains sont plutôt de tendance conservatrice. Ils ont la nostalgie du passé. Il n'y a que peu de radicaux parmi eux. D'une façon générale, la politique les dégoûte, même s'ils votent démocrate de génération en génération. Si l'on n'est pas surpris que beaucoup de cols bleus demeurent à la maison le jour de l'élection, il est étonnant qu'un aussi grand nombre participent aux scrutins.

Mais ceux qui votent, par quoi sont-ils influencés? Catholiques pour la plupart, ils auront tendance à appuyer le parti démocrate qui a été le véhicule principal des principes de la sociologie chrétienne en matière politique. Restés fidèles à leur groupe ethnique, les cols bleus voteront de préférence pour des candidats de leur propre origine. Dans leur appréciation des hommes politiques, les ouvriers manuels se laisseront attirer beaucoup plus par la personnalité des candidats que par l'analyse objective et critique des programmes proposés. Enfin, pour eux tous les politiciens sont du pareil au même et ils prennent pour acquis que la politique est inévitablement corrompue et qu'il y a bien peu d'hommes publics capables de résister à la tentation.

D'une façon générale, le comportement des cols bleus indique qu'ils sont de tendance conservatrice, favorables à une autorité forte, peu enclins, sauf quelques rares exceptions, à prêter attention aux appels des radicaux et des intellectuels. En fait, par exemple, c'est dans leur catégorie sociale qu'on trouve les positions les plus fortement anti-communistes et anti-socialistes et c'est parmi eux également que se recrute une bonne partie des opposants aux noirs, à preuve l'appui qu'ils ont donné au cours des dernières années à George Wallace et leur participation importante aux cellules du Klan. Bref, ils sont naturellement racistes.

Sur un plan plus général, on se rend compte que les générations actuelles de cols bleus n'ont guère d'esprit de classe et la solidarité ouvrière, dont les chefs syndicaux américains aiment parfois de gargariser, n'est pas pour demain. Au contraire, la plupart d'entre eux compte sur leurs propres moyens, pour atteindre un niveau social plus élevé, sinon pour eux-mêmes, du moins pour leurs enfants. Ils restent donc dans l'ensemble résolument optimistes quant à l'avenir.

Et pourtant leur situation n'est pas tellement reluisante. On le voit bien dans le chapitre consacré aux conditions de santé de la classe ouvrière. D'une part, les statistiques révèlent que c'est dans cette catégorie de travailleurs que le taux d'absentéisme pour cause de maladie est le plus haut et, d'autre part, c'est elle qui dépense le moins par personne pour les soins médicaux, entre $\$ 125$. et $\$ 104$. par année contre $\$ 169$. pour la catégorie des cols blancs. D'une façon générale, le col bleu redoute le recours au service du médecin et préfère s'en tenir aux médicaments empiriques ou tout simplement verser dans le fatalisme, ce qui s'expliquerait, selon l'auteur, par l'influence des origines ethniques de beaucoup d'entre eux. Le col bleu s'extasie, par exemple, devant les progrès de la médecine contemporaine et considère souvent l'homme de la science comme un superhumain, mais il craint de l'approcher.

Le coût des soins médicaux est aussi un obstacle à leur généralisation dans la classe ouvrière, mais Shostak rend ici hommage aux syndicats qui ont bataillé ferme pour éliminer graduellement la contribution des salariés dans les régimes d'assurance-maladie et pour obtenir des régimes plus complets. Un point particulier à noter: l'auteur entrebaille une porte en jetant un coup d'oeil sur la santé mentale des cols bleus. Se référant à des études systématiques, il signale que le nombre des déficients mentaux est plus grand dans la classe ouvrière que dans les autres catégories sociales et, ce qui est plus grave, que le processus de détérioration s'accélère sans cesse. Cet état de chose serait attribuable à l'isolement des membres de la famille les uns des autres, à l'ivrognerie, au taux élevé des divorces et des séparations. Raison profonde: la difficulté qu'a le col bleu à s'exprimer et à extérioriser ses sentiments à cause du comportement des employeurs à son endroit, comportement fait d'indifférence et parfois de mépris, à cause des luttes intenses qu'il doit soutenir pour obtenir et conserver un emploi; à cause du travail routinier qui porte au repliement 
et à la rêverie, à cause aussi d'un travail routinier qu'il accomplit seul à coeur de jour, sans aucun contact humain, toutes choses qui engendrent, au niveau du foyer, de graves conflits du silence.

Les syndicats ont également réagi contre cette situation en tentant d'insérer dans les régimes d'assurance-maladie les traitements psychiâtriques. Mais c'est là un côté plutôt négatif; c'est vers le réaménagement du milieu de travail qu'il faudrait tendre.

Bref, en matière de santé, des progrès ont été réalisés, mais, à cause des attitudes fatalistes du travailleur manuel en général, il reste beaucoup à faire, principalement en matière d'hygiène et de médecine préventive.

Dans un chapitre final, l'auteur traite tour à tour du comportement religieux et du problème de la retraite chez le col bleu. La très grande majorité des travailleurs manuels adhèrent à la religion catholique ou à l'une ou l'autre des principales Eglises protestantes, quoique les sectes religieuses $y$ recrutent aussi, principalement chez ceux que la vie a particulièrement malmenés, un certain nombre d'adeptes.

La plupart des ouvriers ne voient pas dans la religion un moyen d'entretenir en eux l'humilité ou l'inquiétude de conscience. Au contraire, la religion est considérée comme la garantie que tout ce qui est américain est valable. La religion, pour l'ouvrier, c'est la validation de ses buts, de ses idéaux plutôt que leur remise en question. La religion offre le salut à l'intérieur de limites praticables bien plus qu'elle n'exige une foi aveugle. Dans la vie quotidienne, la religion sert d'étalon critique. Où faut-il vivre ? Avec qui convient-il de lier amitié ? Qui faut-il marier ?

En matière religieuse tout comme dans les questions politiques, le col bleu est facilement réactionnaire. C'est chez les ouvriers catholiques que les réformes liturgiques récentes ont trouvé le plus de résistance et que les théologiens modernes aux idées un peu avancées sont le plus mis en doute. Ils n'aiment pas que le clergé se mêle aux affaires politiques et sociales. Enfin, l'Auteur, à tort ou à raison, voit dans l'attitude de l'Eglise catholique à l'endroit de la procréation dirigée une des causes de la diminution de la pratique religieuse en milieu ouvrier à cause du sentiment de culpabilité qui en découle et des éléments de division qu'il entraîne dans les foyers.
Et voici le col bleu arrivé à l'âge de la retraite : la plupart du temps, il cessera de travailler vers l'âge de 65 ans, mais il ne le fera pas de gaieté de coeur. Une enquête qui remonte à une dizaine d'années indiquait que 28 pour cent seulement se retirait pour jouir de leurs loisirs, aider à la maison ou échapper à la monotonie du travail. Les autres y étaient forcés par leur état de santé, un régime de retraite obligatoire ou l'impossibilité de trouver du travail. A ce moment, pour le grand nombre, c'est l'isolement et la solitude, un état de santé qui les tient rivés sur place ou une situation économique voisine de la pauvreté, même si les retraités sont généralement moins endettés que les autres. L'Auteur conclut mélancoliquement : le retraité découvre soudain que la vie qu'il n'a pas vécue plus tôt, il ne pourra la reprendre la vieillesse venue.

Quelles conclusions 1'Auteur tire-t-il de son analyse? En d'autres mots, quelles sont les perspectives d'avenir pour le col bleu de race blanche aux Etats-Unis? Quelles sont les améliorations souhaitables? Shostak ne s'étend pas beaucoup sur le sujet et ses suggestions, peu nombreuses, peuvent étonner à cause de leur nature même : multiplier les cliniques de planification familiale et les garderies d'enfants, modifier les structures du système d'éducation de manière que les fils et les filles de familles ouvrières puissent atteindre à un niveau de culture valable, instituer des organismes auxquels les cols bleus pourront s'adresser pour se démêler dans l'inextricable dédale des services administratifs publics et privés, réaménager le régime industriel de façon à établir des unités de production plus petites afin de redonner au travailleur le sens du travail et à diminuer les distances qui séparent les usines du foyer et apporter des réformes au régime actuel de santé physique et mentale. Pour l'Auteur, il ne s'agit pas là de moyens superficiels, de trucs, car même si les réformes prises isolément ne peuvent résoudre les problèmes des travailleurs manuels, toutes peuvent y aider, chacune à leur façon.

De l'ouvrage de Shostak, on peut dire qu'il est une étude en profondeur de la situation globale du col bleu américain. Il suffit, pour en être sûr, de signaler que, sur 300 pages, une cinquantaine sont consacrées à rendre compte de la documentation, livres, revues, rapports, qu'il a colligée pour couvrir son sujet. 
Le lecteur qui s'efforce, en parcourant ce livre, d'appliquer au contexte canadien les constatations et les observations de l'Auteur, y découvrira plusieurs points de ressemblance avec la situation des travailleurs manuels au Canada. Enfin, malgré leur poids imposant dans la collectivité américaine, il n'apparaît pas que les cols bleus puissent y être un ferment de révolution, bien au contraire. $\mathrm{Et}, \mathrm{si}$ un changement intervenait, ce serait plutôt sous la forme d'un écroulement par l'intérieur!

\section{André ROY}

Répertoire des grèves dans la province de Québec au XIXe siècle, par Jean Hamelin, Paul Laroque et Jacques Rouillard. Montréal, Les Presses de l'Ecole des Hautes Etudes Commerciales, $1970,168 \mathrm{pp}$.

A partir d'un inventaire systématique des journaux de l'époque, les auteurs ont fait un relevé des grèves qui ont eu lieu dans la province de Québec au cours du XIXe siècle. (En fait, la première qui est mentionnée date de 1843). Elles sont schématiquement présentées dans un ordre chronologique avec la date, la durée, le lieu, la branche d'activité, le nombre de grévistes, le syndicat concerné, le litige et le règlement. Lorsqu'il s'agit d'une grève longue, un récit du déroulement de la grève dégage les faits saillants. On y donne aussi des indications bibliographiques.

$\mathrm{Ce}$ sont, en somme, des fiches de travail que les auteurs ont réunies et qu'ils mettent à la disposition des chercheurs qui voudront écrire l'histoire des conflits du travail au Québec durant cette période. Comme on s'est basé uniquement sur les journaux, il manque de données importantes et il se glisse des imprécisions que seules des études plus poussées dans les archives publiques et privées pourront combler. Il est encore regrettable que l'on n'ait pas pris la peine de fournir les références aux travaux qui ont déjà été publiés sur certaines grèves particulières.

Néanmoins, en plus d'être un instrument de travail précieux pour les chercheurs, cet ouvrage, malgré son schématisme et ses imperfections, est déjà intéressant à parcourir, car il lève un peu le coin du voile sur certaines mani- festations de la lutte ouvrière au Québec au cours de cette période où l'industrialisation n'était pas encore très poussée.

Gérard DION

Labor Economics, by C.G. Williams, Toronto, John Wiley \& Sons, 1970, 489 pp.

Le présent ouvrage couvre en gros les mêmes sujets que la plupart des autres volumes du même titre. Ces sujets se regroupent sous deux thèmes principaux. Dans le premier, soit l'aspect économique du marché du travail, les auteurs traitent de l'offre et de la demande du travail, de même que de la rémunération et de façon plus spécifique des différentiels de salaire. Dans le second, soit l'aspect institutionnel du même marché, ils font voir l'impact des syndicats et des gouvernements sur le processus de la négociation collective, ou tout simplement sur les relations entre employeurs et employés.

Cependant, certains ouvrages sont plus théoriques, d'autres plus descriptifs. Le présent ouvrage se classe parmi ceux du dernier type. Mais tout en étant descriptif dans son approche globale, l'auteur tente régulièrement d'analyser ou d'expliquer les principaux phénomènes observés. De plus, cette analyse s'appuie sur des études sérieuses et pour la majorité d'entre elles très récentes.

Bien entendu, un tel ouvrage descriptif présente un intérêt immédiat plus grand pour le lecteur américain que pour le lecteur étranger. Il faut quand même garder à l'esprit que le fonctionnement du marché du travail au Canada ressemble passablement à celui du marché américain, à l'exception probablement du domaine législatif.

Tout compte fait, le lecteur canadien peut certes bénéficier grandement de la lecture du volume de Williams. Ceci est d'autant plus vrai que le texte se situe continuellement au même niveau de difficulté. Il convient davantage ici de parler de niveau de facilité, car l'âuteur ne s'adresse définitivement pas d'abord à l'économiste professionnel, mais plutôt à un public plus vaste comprenant, par exemple, les étudiants prégradués.

Nous recommandons la lecture de Labor Economics écrit par C.G. Wil- 